Stortbeker, I. , Houwen, J., Dulmen, S. van, olde Hartman, T, Das, E. Quantifying implicit uncertainty in primary care consultations: Asystematic comparison of communication about medically explained versus unexplained symptoms. Patient Education and Counseling: 2019, 102, p. 2349-2352

$\begin{array}{lll}\text { Postprint version } & : & 1.0 \\ \text { Journal website } & : & \text { https://www.sciencedirect.com/science/article/abs/pii/S0738399119302 } \\ & & 629 ? v i a \% 3 D i h u b \\ \text { Pubmed link } & : & \text { https://www.ncbi.nlm.nih.gov/pubmed/31288956 } \\ \text { DOI } & : & 10.1016 / j . p e c .2019 .07 .005\end{array}$

This is a Nivel certified Post Print, more info at nivel.nl

\title{
Quantifying implicit uncertainty in primary care consultations: A systematic comparison of communication about medically explained versus unexplained symptoms
}

\author{
Inge Stortenbeker ${ }^{a, *}$, Juul Houwen ${ }^{b}$, Sandra van Dulmen ${ }^{b, c, d}$, Tim olde \\ Hartman $^{\text {bee }}$, Enny Das ${ }^{\mathrm{a}}$
}

a Centre for Language Studies, Radboud University, Nijmegen, The Netherlands

b Radboud university medical center, Radboud Institute for Health Sciences, Department of Primary and Community Care, Nijmegen, The Netherlands

c NIVEL (Netherlands institute for health services research), Utrecht, The Netherlands

d Faculty of Health and Social Sciences, University of South-Eastern Norway, Drammen, Norway

e Donders Institute for Brain, Cognition and Behaviour, Nijmegen, The Netherlands

\begin{abstract}
A B S T R A C T
Objective: General practitioners (GPs) disclose more uncertainty (e.g. "I don't know") in consultations with patients presenting medically unexplained symptoms (MUS) versus medically explained symptoms (MES), which could negatively affect patient outcomes. This study assessed if this pattern also holds for more subtle, implicit uncertainty expressions (e.g. "maybe", "might") during different consultation phases, and assessed their relation to patient pre-post consultation anxiety.

Methods: We quantified implicit markers of uncertainty of 18 GPs in 82 consultations about MUS or MES during different consultation phases. Relative frequencies of implicit uncertainty per consultation were regressed on differences in momentary anxiety pre and post consultation.

Results: We coded $2590 \mathrm{GP}$ utterances. Uncertainty expressions were more frequent in MUS versus MES consultations ( $O R=1.54, p=.004)$, especially during diagnosis and treatment recommendations compared to physical examinations (OR $=0.45, p=.001$ ). Implicit uncertainty was not related to patients' changes in anxiety $(b=0.11, p=.817)$. Conclusions: GPs express more uncertainty during MUS (versus MES) consultations, especially during the diagnostic phase and treatment recommendations. This does not necessarily affect patient anxiety.
\end{abstract}


Stortbeker, I. , Houwen, J., Dulmen, S. van, olde Hartman, T, Das, E. Quantifying implicit uncertainty in primary care consultations: Asystematic comparison of communication about medically explained versus unexplained symptoms. Patient Education and Counseling: 2019, 102, p. 2349-2352

Practice implications: Implicit uncertainty expressions reflect the mere complexity of communicating a medically unexplained diagnosis, which does not affect patient anxiety.

\section{Introduction}

Uncertainty is inherent to medicine [1,2]. Physicians verbally disclose uncertainty in nearly threequarters of medical encounters [3]. Especially when patients present symptoms with no detectable underlying cause, i.e. when they present medically unexplained symptoms (MUS), a physician's message inevitably has an element of uncertainty [4]. Physicians state their uncertainty more often when patients have MUS (e.g. vague chestpain) compared to their medically explained counterpart (e.g. chest pain due to acid reflux; i.e. medically explained symptoms (MES)) [5].

Previous research has operationalized the extent to which uncertainty is disclosed in medical consultations by including explicit statements such as "I don't know" [5], "it is not clear" [3], and "I need to find out more" [6]. There are, however, other, implicit strategies to express uncertainty in a more subtle manner, e.g. with certain adverbs (e.g. "probably", "maybe"), modal verbs ("might", "may"), and conditional phrases ("if you feel better in a week") [7]. Uncertainty statements such as "I don't know" may negatively affect patient satisfaction with a consultation or physician, because patients infer that their physician is not competent [8,9], though findings are mixed [10]. However, implicit uncertainty expressions may also reflect physicians' formulation effort [11] during medical interactions about MUS. Rather than producing negative effects, such implicit uncertainty expressions may facilitate talk about delicate issues, though researchers pointed to potential downsides such as inviting patients' resistance [11].

Previous analysis of implicit uncertainty in MUS consultations is based on qualitative analyses [11]. Whether implicit uncertainty is specific for MUS consultations is yet unknown, because to date a systematic quantitative analysis of medical interactions is lacking. This research therefore aimed to extend previous qualitative findings by quantifying general practitioners' (GPs) implicit uncertainty expressions for MUS and MES consultations during different phases of the consultation, and explored its relation to patient anxiety. Based on previous findings [5], we hypothesized that implicit uncertainty would be more prominent in MUS (versus MES) consultations, across different phases of the consultation [11]. Furthermore, we looked into the potential effects of GP's implicit uncertainty expressions no changes in patient anxiety post consultation.

\section{Methods}

\subsection{Participants and procedure}

This study was part of a larger research on GP-patient communication about MUS in which 393 everyday consultations of 20 GPs were video-recorded on 1-2 days per GP. Forty-one consultations were labelled as MUS consultation by $18 \mathrm{GPs}[12,13]$. To compare uncertainty of GPs, each MUS consultation was compared to a MES consultation (same GP, same day for all except three consultations) resulting in a dataset of 82 consultations. Patient's age (49 female) varied from 18 to 86 years $(M=52.6 ; S D=17.9)$. All patients filled in a pre- and post-consultation questionnaire including the short version of the State Trait Anxiety Inventory (STAI) [14] with ten statements (1 = "not at all", 4 = "very much so") assessing their state anxiety (range: 1.00-3.80, difference score range: 1.40-1.37; higher scores indicated higher anxiety levels).

\subsection{Coding procedure and reliability}

Two coders identified and categorized GPs' expressions about patients' medical situation. We describe the coding procedure in more detail elsewhere [13]. Frequent expressions of implicit 
Stortbeker, I. , Houwen, J., Dulmen, S. van, olde Hartman, T, Das, E. Quantifying implicit uncertainty in primary care consultations: Asystematic comparison of communication about medically explained versus unexplained symptoms. Patient Education and Counseling: 2019, 102, p. 2349-2352

uncertainty such as uncertain verbs (e.g. "could", "I think"), lexical items (e.g. "probably", "maybe") and pragmatic particles (e.g. "sort of") were coded (Krippendorff's $\alpha=0.77,95 \% \mathrm{Cl} 0.62-0.90$ ) $[15,16]$. The consultation phase of these expressions was also coded (physical examination, diagnosis, or treatment recommendation; Krippendorff's $\alpha=0.71,95 \% \mathrm{Cl} 0.63-0.79$ ).

\subsection{Data analysis}

We used binary logistic mixed-effects models to predict variation in uncertainty expressions across consultations with patients with MUS and MES, and we used a linear mixed-effects model to test the relationship between the relative frequency of uncertainty per consultation (uncertain expressions +1 / all relevant expressions +1 ) and difference scores of patient anxiety. The data were analysed using $\mathrm{R}$ with the Ime4 package.

\section{Results}

We identified 2590 expressions about patients' medical situation. Table 1 presents the distribution of implicit uncertainty expressions per phase of the consultation for patients with MUS and MES.

\section{[Table 1]}

We first specified an empty model, i.e. a model without predictor variables for random intercepts with the best model fit $\left(A I C=3067.5, X^{2}(3)=8.94, p=.003\right)$. There was significant variance in intercepts across GPs (variance $=.05, S D=0.23$ ), and patients (variance $=.07, S D=.26$ ). We added potential predictors to the empty model. "Type of complaints (MUS or MES)", "consultation phase", and the interaction term between the two variables significantly improved the model fit (AIC = $3057.0, X^{2}(8)=19.89, p=.002$ ). Potential confounding variables (GP sex or age; patient sex, age, educational level, work status, or repeated visit for symptoms; word total per consultation) or random slopes did not contribute to the model fit. As can be observed in Table 2 and Fig. 1, GPs were 1.54 times more likely to express uncertainty when patients had MUS compared to MES.

Furthermore, whereas uncertainty expressions were significantly higher for the diagnostic phase and treatment recommendations than the physical examination phase in MUS consultations $(O R=0.45, p$ $=.001)$, this difference was absent for MES consultations (OR $=1.07, p=.785)$. We related indices of uncertainty per consultation to patient anxiety. There was a significant variance in intercepts across GPs (variance $=0.02, S D=0.15$ ). No significant random slopes or confounding variables improved the fit of the model. We observed no significant relationship between the amount of uncertainty expressed by GPs, and the pre-post consultation difference in anxiety of all patients (MUS and MES) $(b=0.11, \mathrm{SE}=0.49, p=.817,95 \% \mathrm{Cl}-1.19-0.91)$.

\section{Discussion and conclusion}

\subsection{Discussion}

This study was the first to quantitatively analyse GPs' implicit expressions during consultations about MUS versus MES, and their relationship with patients' pre-post consultation difference in anxiety. GPs expressed more implicit uncertainty in consultations about MUS compared to MES, especially during the phase of diagnosis and treatment recommendations. This indicates that, apart from explicit uncertainty expressions such as "I don't know" [5], GPs also systematically express more implicit uncertainty in consultations about MUS. These findings provide a quantitative extension of previous qualitative studies reporting a high formulation effort (e.g. self-corrections, reformulations) in MUS consultations, especially during diagnosis and treatment recommendations [11]. We demonstrate that this only holds for MUS but not MES consultations. The increase in implicit uncertainty may be attributed to the fact that GPs can rely on their professional knowledge when 
Stortbeker, I. , Houwen, J., Dulmen, S. van, olde Hartman, T, Das, E. Quantifying implicit uncertainty in primary care consultations: Asystematic comparison of communication about medically explained versus unexplained symptoms. Patient Education and Counseling: 2019, 102, p. 2349-2352

physically examining a patient, while diagnosis and treatment recommendations for MUS are made within the physician-patient dialogue. This could contribute to increased uncertainty expressions during those phases of the consultation.

Although it was previously shown that linguistic elements (directness of positive and negative messages) may affect patients' state anxiety [13], our findings show no relationship between implicit uncertainty and patient anxiety. This contributes to previous findings about uncertainty and patient anxiety [8], which has reported both positive and negative effects of uncertainty expressions on patient satisfaction $[3,7,9]$. Rather than reflecting physicians' professional insecurity, implicit uncertainty may reflect the complex reality of communicating a medically unexplained diagnosis. It could be a way to search for the right label when medical explanatory models do not suffice to explain symptoms [17], which need not affect patient anxiety after the consultation.

\section{[Table 2] [Figure 1]}

Some limitations of the study need to be addressed in future research. We did not compare effects of different types of uncertainty expressions, which could also explain a lack of association between implicit uncertainty and patient anxiety. Future studies should focus on comparing different types of uncertainty expressions (e.g. "I don't know" vs. "maybe"), and their relationship with patient responses, taking into account potential cultural differences that may underlie the interpretation of uncertainty expressions [18]. Further analyses should take into account the sequentiality of these interactions (i.e. how patients respond, e.g. remaining silent or minimal responses [11]) to examine how this could affect physician implicit uncertainty expressions. Finally, we compared implicit uncertainty expressions to patients' self-reported anxiety measures. Future research should compare effects of implicit uncertainty expressions for different anxiety measures (e.g. tension scale of Profile of Mood states [19]), and for different patient outcomes such as patient satisfaction.

\subsection{Conclusion}

This research extends previous findings by providing quantitative evidence that the number of physicians' implicit uncertainty expressions differs between MUS (versus MES) conversations, and is most prevalent during the diagnostic phase and treatment recommendations. Implicit uncertainty expressions do not necessarily affect changes in patient anxiety.

\subsection{Practice implications}

Doctors may affect patient outcomes when they explicitly express their uncertainty (i.e. "I don't know"). We demonstrate that expression of more subtle, implicit uncertainty such as "I think your headache may have two causes" does not affect post-consultation anxiety. Rather, implicit uncertainty expressions such as "maybe" or "it could be" reflect the mere complexity of communicating a diagnosis that has no medical explanation. GPs should not worry about (unconsciously) revealing this complexity during the medical interaction.

\section{Ethical approval and consent to participate}

The data obtained for this study and additional analyses have been approved by the Ethics Committee of the Radboud University Medical Centre (file number2015-1566). A written informed consent was obtained from all participants before entry into the study.

\section{Funding}

The data collection was supported by the Dutch public funding agency ZonMw (grant number 839110010); and analysis and interpretation of the data was supported by Netherlands Organisation for Scientific Research NWO (grant number PWG.17.031). 
Stortbeker, I. , Houwen, J., Dulmen, S. van, olde Hartman, T, Das, E. Quantifying implicit uncertainty in primary care consultations: Asystematic comparison of communication about medically explained versus unexplained symptoms. Patient Education and Counseling: 2019, 102, p. 2349-2352

References

[1] A.K. Ghosh, Understanding medical uncertainty: a primer for physicians, J. Assoc. Physicians India 52 (2004) 739-742.

[2] C. Wellbery, The value of medical uncertainty? Lancet 375 (9727) (2010) 1686-1687, doi:http://dx.doi.org/10.1016/S0140-6736(10)60725-8.

[3] G.H. Gordon, S.K. Joos, J. Byrne, Physician expressions of uncertainty during patient encounters, Patient Educ. Couns. 40 (1) (2000) 59-65, doi:http://dx.doi.org/10.1016/S0738-3991(99)000695.

[4] L.A. Page, S. Wessely, Medically unexplained symptoms: exacerbating factors in the doctorpatient encounter, J. R. Soc. Med. 96 (5) (2003) 223-227, doi: http://dx.doi.org/10.1177/014107680309600505.

[5] R.M. Epstein, T. Hadee, J. Carroll, S.C. Meldrum, J. Lardner, C.G. Shields, Could this be something serious?" Reassurance, uncertainty, and empathy in response to patients' expressions of worry, J. Gen. Intern. Med. 22 (12) (2007) 1731-1739, doi:http://dx.doi.org/10.1007/s11606-007-0416-9.

[6] J. Ogden, K. Fuks, M. Gardner, S. Johnson, M. McLean, P. Martin, R. Shah, Doctors expressions of uncertainty and patient confidence, Patient Educ. Couns. 48 (2) (2002) 171-176, doi:http://dx.doi.org/10.1016/S0738-3991(02)00020-4.

[7] G. Cousin, M. Schmid Mast, N. Jaunin-Stalder, When physician-expressed uncertainty leads to patient dissatisfaction: a gender study, Med. Educ. 47 (9) (2013) 923-931, doi:http://dx.doi.org/10.1111/medu.12237.

[8] D.C. Blanch, J.A. Hall, D.L. Roter, R.M. Frankel, Is it good to express uncertainty to a patient? Correlates and consequences for medical students in a standardized patient visit, Patient Educ. Couns. 76 (3) (2009) 300-306, doi: http://dx.doi.org/10.1016/j.pec.2009.06.002.

[9] C.G. Johnson, J.C. Levenkron, A.L. Suchman, R. Manchester, Does physician uncertainty affect patient satisfaction? J. Gen. Intern. Med. 3 (2) (1988) 144-149.

[10] R. McGovern, D. Harmon, Patient response to physician expressions of uncertainty: a systematic review, Ir. J. Med. Sci. 186 (4) (2017) 1061-1065, doi: http://dx.doi.org/10.1007/s11845-0171592-1.

[11] C.M. Monzoni, R. Duncan, R. Grunewald, M. Reuber, How do neurologists discuss functional symptoms with their patients: a conversation analytic study, J. Psychosom. Res. 71 (6) (2011) 377-383, doi:http://dx.doi.org/10.1016/ j.jpsychores.2011.09.007.

[12] J. Houwen, P. Lucassen, H.W. Stappers, P.J.J. Assendelft, S. van Dulmen, T.C. Olde Hartman, Medically unexplained symptoms: the person, the symptoms and the dialogue, Fam. Pract. 34 (2) (2017) 245-251, doi:http://dx.doi.org/10.1093/ fampra/cmw132.

[13] I.A. Stortenbeker, J. Houwen, P.L.B.J. Lucassen, H.W. Stappers, W.J.J. Assendelft, S. van Dulmen, T.C. Olde Hartman, E. Das, Quantifying positive communication: doctor's language and patient anxiety in primary care consultations, Patient Educ. Couns. 101 (9) (2018) 1577-1584, doi:http://dx. doi.org/10.1016/j.pec.2018.05.002.

[14] C.D. Spielberger, R.L. Gorsuch, P.R. Lushene, P.R. Vagg, G.A. Jacobs, Manual for the State-Trait Anxiety Inventory, Consulting Psychologists Press, Inc., 1983.

[15] M. Green, Cognitive stage differences in types of speaker uncertainty markers, Lang. Speech 27 (October) (1984) 323-331, doi:http://dx.doi.org/10.1177/ 002383098402700403.

[16] E.T. Vold, Epistemic modality markers in research articles: a cross-linguistic and crossdisciplinary study, Int. J. Appl. Linguist. 16 (1) (2006) 61-87, doi: http://dx.doi.org/10.1111/ j.1473-4192.2006.00106.x.

[17] M.L. Johansen, M.B. Risor, What is the problem with medically unexplained symptoms for GPs? A meta-synthesis of qualitative studies, Patient Educ. Couns. 100 (4) (2017) 647-654, doi:http://dx.doi.org/10.1016/j. pec.2016.11.015. 
Stortbeker, I. , Houwen, J., Dulmen, S. van, olde Hartman, T, Das, E. Quantifying implicit uncertainty in primary care consultations: Asystematic comparison of communication about medically explained versus unexplained symptoms. Patient Education and Counseling: 2019, 102, p. 2349-2352

[18] T.S. Doupnik, M. Richter, Interpretation of uncertainty expressions: a cross-national study, Account. Organ. Soc. 28 (1) (2003) 15-35, doi:http://dx.doi.org/10.1016/S0361-3682(02)000107.

[19] S. Shacham, A shortened version of the Profile of Mood States, J. Pers. Assess. 47 (3) (1983) 305-306, doi:http://dx.doi.org/10.1207/s15327752jpa4703_14.

\section{Tables and figures}

Table 1. Distribution of uncertainty expressions in GPs' statements per phase of the consultation for consultations with patients with MUS $(n=1480)$ and MES $(n=1110)$.

\begin{tabular}{lll}
\hline & MUS & MES \\
$n(\%)$ & $n(\%)$ \\
\hline Total & $439(29.7)$ & $295(26.6)$ \\
Physical examination & $29(18.4)$ & $34(24.5)$ \\
Diagnosis & $228(30.9)$ & $100(24.3)$ \\
Treatment recommendations & $182(31.1)$ & $161(28.8)$ \\
\hline
\end{tabular}

Table 2. Final binomial mixed model of GP expressed implicit uncertainty.

\begin{tabular}{|c|c|c|c|c|c|c|}
\hline & Estimate & $S E$ & OR & z-value & $\operatorname{Pr}(>\mid z)$ & $95 \% \mathrm{CI}$ \\
\hline Intercept & -1.20 & 0.14 & 0.30 & -8.50 & & \\
\hline MUS vs. MES & 0.44 & 0.15 & 1.54 & 2.56 & .004 & $1.15-.2 .10$ \\
\hline Contrast 1: $\mathrm{PE}^{\mathrm{a}} \mathrm{vs}$. diagnosis and treatment & 0.05 & 0.23 & 1.06 & 0.24 & .812 & $0.66-1.68$ \\
\hline Contrast 2: Treatment vs. PE and diagnosis & 0.21 & 0.15 & 1.23 & 1.35 & .178 & $0.91-1.68$ \\
\hline MUS vs. MES * contrast 1 & -0.86 & 0.33 & 0.42 & -2.61 & .009 & $0.22-0.81$ \\
\hline MUS vs. MES * contrast 2 & -0.28 & 0.20 & 0.76 & 1.83 & .167 & $0.51-1.12$ \\
\hline
\end{tabular}

a $\mathrm{PE}=$ physical examination.

Figure. 1. Predicted probabilities with standard errors of implicit uncertainty expressions for type of complaints (MUS vs. MES) and phases of the consultation

\section{Predicted probabilities of GP uncertainty expressions}

\section{MUS}

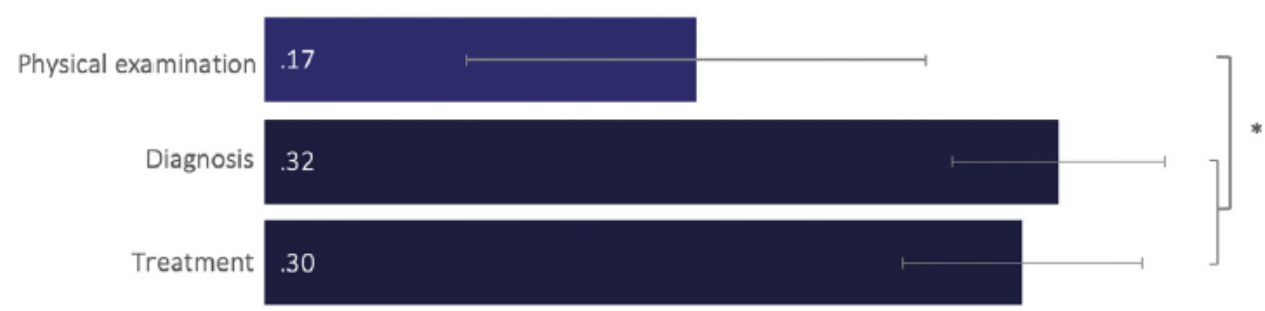

\section{MES}

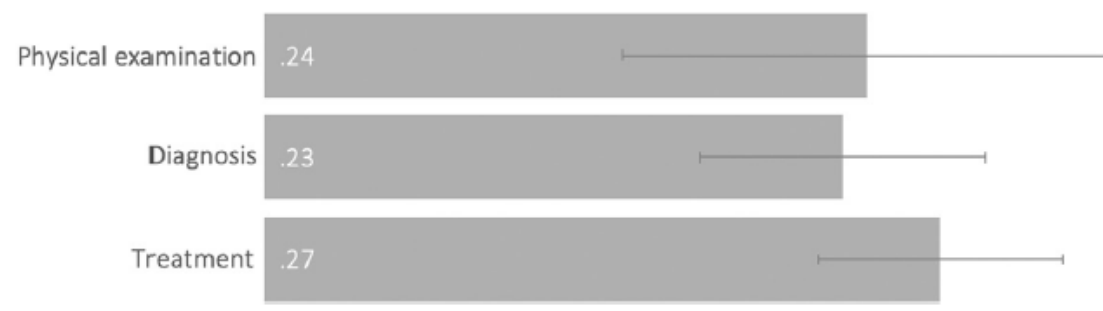

